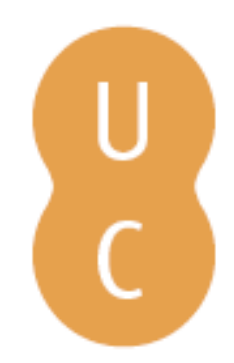

\title{
pompalina
}

La notion de philanthropia chez Plutarque: contexte social et sources philosophiques

Autor(es): Becchi, Francesco

Publicado por: Imprensa da Universidade de Coimbra; Centro de Estudos Clássicos e

URL

persistente: URI:http://hdl.handle.net/10316.2/32004

DOI: $\quad$ DOI:http://dx.doi.org/10.14195/978-989-8281-17-3_24

Accessed : $\quad$ 26-Apr-2023 12:27:30

A navegação consulta e descarregamento dos títulos inseridos nas Bibliotecas Digitais UC Digitalis, UC Pombalina e UC Impactum, pressupõem a aceitação plena e sem reservas dos Termos e Condições de Uso destas Bibliotecas Digitais, disponíveis em https://digitalis.uc.pt/pt-pt/termos.

Conforme exposto nos referidos Termos e Condições de Uso, o descarregamento de títulos de acesso restrito requer uma licença válida de autorização devendo o utilizador aceder ao(s) documento(s) a partir de um endereço de IP da instituição detentora da supramencionada licença.

Ao utilizador é apenas permitido o descarregamento para uso pessoal, pelo que o emprego do(s) título(s) descarregado(s) para outro fim, designadamente comercial, carece de autorização do respetivo autor ou editor da obra.

Na medida em que todas as obras da UC Digitalis se encontram protegidas pelo Código do Direito de Autor e Direitos Conexos e demais legislação aplicável, toda a cópia, parcial ou total, deste documento, nos casos em que é legalmente admitida, deverá conter ou fazer-se acompanhar por este aviso.

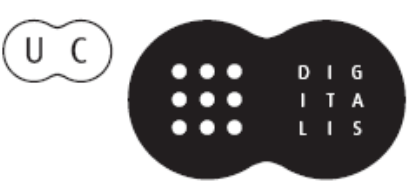




\section{Symposion and Philanthropia in Plutarch}

\section{José Ribeiro Ferreira, Delfim Leão Manuel Troster e Paula Barata Dias (eds.)}

IMPRENSA DA UNIVERSIDADE DE COIMBRA 


\title{
La notion de Philanthrópla Chez Plutarque: CONTEXTE SOCIAL ET SOURCES PHILOSOPHIQUES
}

Francesco Becchi

Université de Florence

\begin{abstract}
In a period in which some natural feelings, like philia and philanthrōpia, are disappearing, it is natural that an intellectual, like Plutarch, asks for the reasons that have determined this disappearance. The philosopher from Chaeronea identifies them in the greed (pleonexia) and in the insatiability (aplestia) that have invaded the soul of the aristocracy of his time. Due to these passions, which derive from wrong judgements and empty opinions, the soul has become attached to goods that are foreign to itself and eventually loses the emotional impulse for showing its proper virtues. Consequently, these passions end up wearing out human relations in such a way as to make man no more familiar and friend to his fellows (oikeios), but a stranger (allotrios).
\end{abstract}

Regarding the philosophical coordinates, Plutarch's philosophy of philanthrōpia as oikeiotés seems to find its starting point in Aristotle's $E N$ and, more in general, in Peripatetic philosophy beginning with Theophrastus.

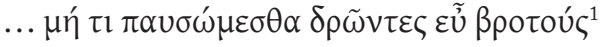

$\mathrm{Au}$ cours des premiers siècles de l'époque impériale, durant lesquels l'éthique païenne et l'éthique chrétienne cohabitent et s'opposent ${ }^{2}$, le sentiment qui domine, est , semble-t-il, celui de la philia pour les biens matériels. La riche aristocratie de cette période, qui est égoïste et égocentrique, est aussi la proie facile des pires passions de l'âme ${ }^{3}$, et apparaît dominée par le désir des richesses ( $\varphi \imath \lambda o x \rho \eta \mu \alpha \tau i ́ \alpha)$, du pouvoir ( $\varphi \imath \lambda \alpha \rho x i ́ \alpha)$ et des honneurs

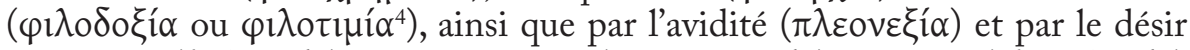

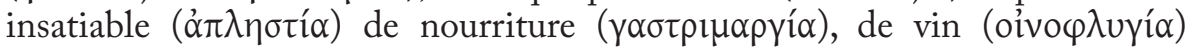
et de sexe $(\lambda \alpha \gamma v \varepsilon i ́ \alpha)^{5}$. Ces passions malsaines de l'âme ont supplanté et fait disparaître de l'esprit humain des sentiments naturels comme la $\varphi \imath \lambda \alpha \delta \varepsilon \lambda \varphi i ́ \alpha$, la $\varphi \imath \lambda o \sigma \tau o \rho \gamma i ́ \alpha$, la $\varphi \imath \lambda \varepsilon \tau \alpha \imath \rho i ́ \alpha$ ou $\varphi \imath \lambda o \varphi \imath \lambda i ́ \alpha$ et la $\varphi \imath \lambda o \xi \varepsilon v i ́ \alpha$ ainsi que la $\varphi \imath \lambda \alpha v \theta \rho \omega \pi i ́ \alpha$ qui avaient caractérisé la civilisation hellénique en général et la

${ }^{1}$ TrGF 2 F 410a Kannicht-Snell, cité par Plutarque dans An Seni resp. 791D et suav. viv. Epic. 1099A.

${ }^{2}$ Voir A. Postiglione dans Plutarco, L'amore fraterno, L'amore per ifigli, Napoli 1991, p. 25: “A1 confine fra l'etica pagana e l'etica cristiana Plutarco dice che bisogna amare tutti gli uomini,... non conoscendo, e tuttavia quasi presagendo quel comandamento più alto, che ormai si andava diffondendo per il mondo, di amare tutti gli uomini come fratelli". Sur ce point il faut rappeler

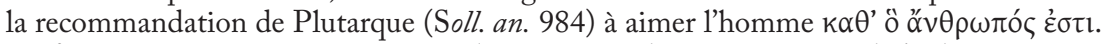

${ }^{3}$ Comme l'envie ou la colère, qui (Coh. ira 462F) est une douleur ( $\lambda$ ú $\pi \eta$ ) et un mélange des semences de toutes les passions ( $\pi \alpha v \sigma \pi \varepsilon \rho \mu i ́ \alpha \tau \tilde{\omega} \nu \pi \alpha \theta \tilde{\omega} v)$.

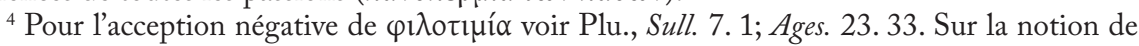
$\varphi \imath \lambda o \tau \imath \mu i ́ \alpha$ chez Plutarque voir maintenant M. C. Fialho, 2008, pp. 45-6.

${ }^{5}$ Voir V. A. Sirago, 1974, pp. 65-83; C. P. Jones, 1978, p. 104 sqq.; P. Desideri, 1978, p. 353 n. 29. 
civilisation athénienne en particulier ${ }^{6}$. Ainsi la dépravation humaine - comme le commente Plutarque, non sans amertume, dans le De fraterno amore ${ }^{7}$ - qui a germé comme la zizanie au milieu du blé ${ }^{8}$, a rendu impossible le fait de trouver un rapport d'amitié qui soit sincère, pur et sans passions 9 . À une époque où la sophistique est en train de devenir prépondérante, il est plus facile de trouver, semble-t-il, quelqu'un capable d'écrire des textes sur l'amitié que quelqu'un qui la mette en pratique ${ }^{10}$. Pourtant l'homme, qui est un être non seulement

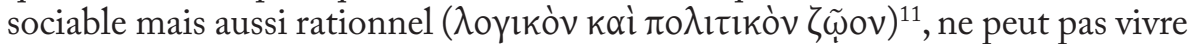

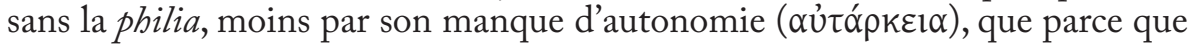
cela est contre nature. Dans la Vie de Solon ${ }^{12}$ Plutarque présente une objection

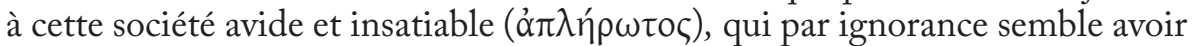
abandonné la nature pour suivre la nature de ce qui est contre nature, avec la conviction que le bonheur consiste à accumuler des richesses et à posséder des biens matériels ${ }^{13}$. Pour lui, l'âme humaine, qui par nature est portée à aimer $(\varphi \imath \lambda \varepsilon \tilde{\imath} v)$, à sentir, à penser, à se souvenir et à apprendre, perd sa charge affective au moment où elle par avidité ou par une ambition excessive perd l'amour pour ce qui lui est propre (oikkeĩov) et apparenté et s'attache aux biens matériels ( $\tau \grave{\alpha}$ $\varepsilon \dot{\varepsilon} \tau o ́ \varsigma)^{14}$. Alors, il est naturel que les rapports humains en soient compromis et que la philanthrōpia disparaisse. Elle perd - pour citer l'introduction de la Vie de Périclès ${ }^{15}$ - le sentiment naturel d'amour et d'affection que l'homme a en lui et qu'il est appelé à manifester à l'égard de ses semblables. Ce sont en effet les soucis dus au désir d'argent qui, entraînant pour l'âme des rides précoces et des

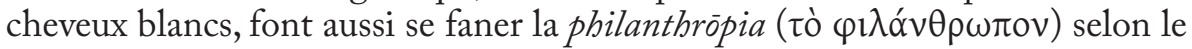
De cupiditate divitiarum ${ }^{16}$.

En confirmant que le bonheur chez l'homme n'arrive pas de l'extérieur et que ce n'est pas quelque chose que l'on peut acheter ${ }^{17}$, le philosophe de Chéronée

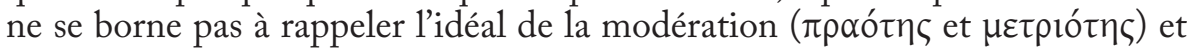

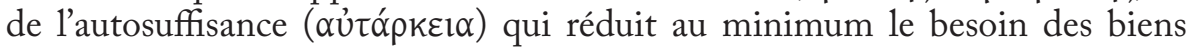

${ }^{6}$ Plu., Frat. am. 478C ; Cup. div. 523D; Comp. Arist. - Cat. Ma. 4.2.

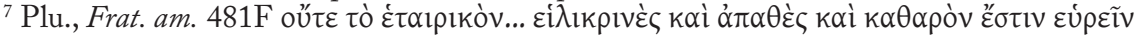
каkíac...

${ }^{8}$ Voir Plu., Am. prol. 497CD.

${ }^{9}$ Plu., Cap. ex inim. ut. 89B ; Luc. 41.9.

${ }^{10}$ Plu., Frat. am. 481BC.

${ }^{11}$ Plu., Am. prol. 495C.

${ }^{12}$ Plu., Sol. 7. 3.

${ }^{13}$ Plu., Cup. div. 524B. On devrait pénser non seulement au petit traité de Plutarque

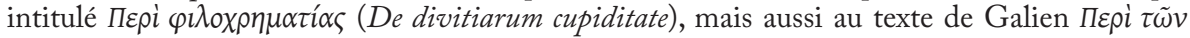

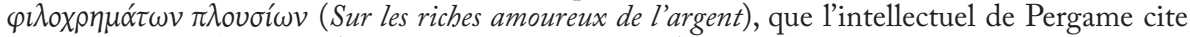

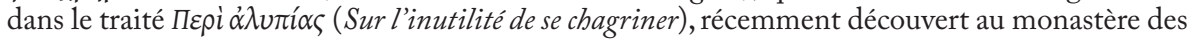
Vlatades à Thessalonique et édité par Véronique Boudon-Millot (V. Boudon-Millot et AL., 2008, pp. 78-123).

${ }_{14}^{14}$ Plu., Sol. 7.3.

${ }^{15}$ Plu., Per. 1. 1-2.

${ }^{16}$ Pour la $\varphi \imath \lambda \alpha \rho \gamma v \rho i ́ \alpha$ qui obscurcie la $\varphi \imath \lambda \alpha v \theta \rho \omega \pi i ́ \alpha$ voir Plu., Cup. div. 526F-527A; Tranq. an. $468 \mathrm{EF}$.

${ }^{17}$ Plu., Fort. 99E; Cap. ex inim. ut. 92DE; Virt. et vit. 100C, 101B-D; Tranq. an. 466D, 477A. 
matériels ${ }^{18}$, mais il montre un nouveau modèle d'humanité aux hommes de son époque, habiles à pratiquer la philia uniquement par les mots ${ }^{19}$. Ce nouveau modèle est caractérisé par la bienveillance et par la bonté, par la générosité et par la clémence, des qualités qui, dans les rapports humains, trouvent un champ d'application plus vaste que celui de la loi et de la justice ${ }^{20}$. Dans la Vie de Caton l'Ancien, en critiquant le comportement dur de Caton à l'égard de ses vieux esclaves, comportement qui est celui d'un homme qui ne pratiquait pas la philanthrōpia mais qui croyait seulement à l'existence de rapports humains fondés sur l'utilité ( $\chi \rho \varepsilon i ́ \alpha)$, Plutarque affirme que tout naturellement l'homme

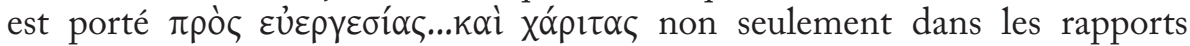
humains, mais aussi vis-à-vis des animaux, si ce n'est pour une autre raison, du moins pour s'exercer à la vertu de la $\varphi \imath \lambda \alpha v \theta \rho \omega \pi i^{21}$.

L'accusation la plus grave que Plutarque adresse à la société de son époque n'est pas seulement celle de la recherche du plaisir ( $\varphi \imath \lambda \eta \delta o v i ́ \alpha)$, de l'avidité insatiable et de la goinfrerie ( $\dot{\alpha} \pi \lambda \eta \sigma \tau i ́ \alpha)$, dues à un jugement faux et irrationnel

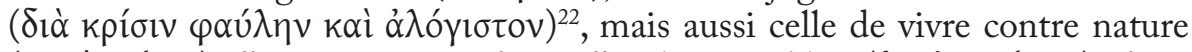

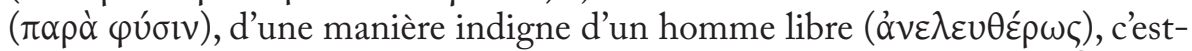
à-dire inhumainement $(\dot{\alpha} \pi \alpha v \theta \rho \omega \hat{\pi} \pi \omega \varsigma)^{23}$, sans jamais rien offrir ( $\left.\alpha \mu \varepsilon \tau \alpha \delta o ́ \tau \omega \varsigma\right)$,

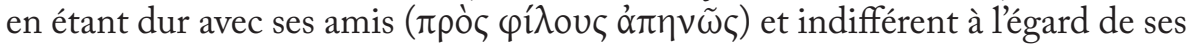

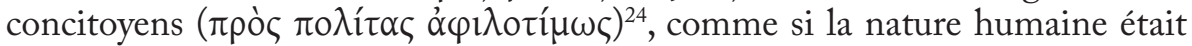
incapable d'aimer de façon désintéressée et sans y trouver son compte ${ }^{25}$. Par contre, parmi les liens sacrés, c'est celui naturel de l'amitié qui est le plus sacré et le plus fort ${ }^{26}$. La conformité avec la nature de ce sentiment de la philia, qui est à la base de tous les rapports humains est démontré par les animaux ${ }^{27}$ qui, ne possédant pas l' adaptabilité, ni l'excellence, ni la pleine autonomie de la raison, suivent leur instinct et demeurent enracinés dans la nature ${ }^{28}$, alors que chez l'homme, la raison qui est la reine absolue et qui se trouve influencée par de nombreuses opinions et de nombreux préjugés, est sortie du droit chemin signalé par la nature et a fini par n'en laisser aucune trace claire et visible ${ }^{29}$.

${ }^{18}$ Plu., Comp. Arist. - Cat. Ma. 4. 2.

${ }^{19}$ Les opuscules qui nous sont parvenus où Plutarque développe le sujet de la $\varphi$ ı $\lambda$ ía sont au nombre de trois: de adulatore et amico, de amicorum multitudine et de fraterno amore. À ceux-ci on

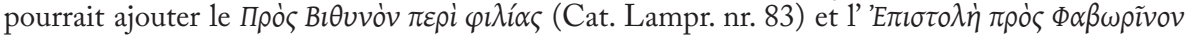

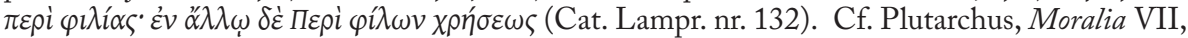
Fragmenta $154-166 \mathrm{Sdb}$.

${ }^{20}$ Plu., Cat. Ma. 5.2.

${ }^{21}$ Plu., Cat. Ma. 5. 2-5.

${ }^{22}$ Plu., Cup. div. 524D.

${ }^{23}$ Cf. Plu., Dio 7. 5. Voir Plu., Frat. am. 479C (’’ $\left.\varphi \imath \lambda \alpha ́ v \theta \rho \omega \pi \circ \varsigma\right)$.

${ }^{24}$ Plu., Cup. div. 525C.

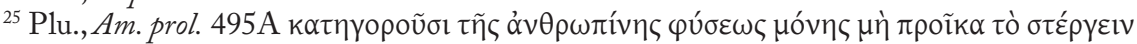

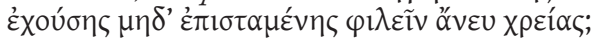

${ }^{26}$ Plu., Frat. am. 479D.

${ }^{27}$ Plu., Am. prol. 493BC.

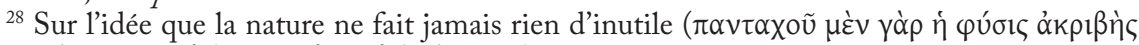

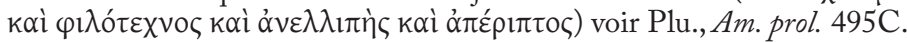

${ }^{29}$ Plu., Am. prol. 493DE. 


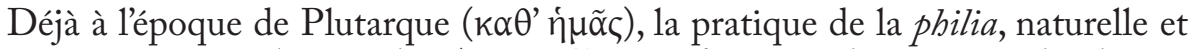
propre à un peuple pour les Anciens ${ }^{30}$, était devenue plus rare que le phénix, rare comme l'avait été la haine chez les Anciens ( $\dot{\varepsilon} \pi i \tau \tilde{\omega} \nu \pi \alpha \lambda \alpha i \tilde{\omega} V)^{31}$; de toute façon, comme nous le confirme Lucien dans son dialogue Sur l'amitié, ce sentiment était depuis longtemps bien loin de pouvoir être considéré comme une exclusivité de la civilisation grecque, celle-ci étant désormais uniquement habile à composer des discours sur l'amitié, mais non plus à la mettre en pratique. Dans le dialogue de Lucien, le scythe Toxaris qui s'adresse à son interlocuteur, le grec Mnésippe, affirme :

Valons-nous mieux que les Grecs sous les autres rapports, sommes-nous plus justes, plus respectueux qu'ils ne le sont envers nos parents ? Je ne prétends pas entrer en contestation avec toi sur cette question. Toujours est-il que les Scythes sont, plus que les Grecs, des amis tendres et fidèles, et que l'amitié est chez nous plus honorée que chez vous : ce serait un point facile à démontrer [...] Vous me paraissez capables de faire sur l'amitié les plus beaux discours du monde ; mais, loin que vos actions répondent à vos paroles, vous vous contentez de la louer et de montrer quel grand bien elle est pour les hommes ; puis, au moment d'agir, traîtres à votre langage, vous fuyez, je ne sais comment, la mise en application de vos théories ${ }^{32}$.

Ainsi, à ses lecteurs qui, comme Caton l'Ancien, ne semblent plus croire à l'existence d'autres rapports que ceux dictés par l'utilitée 33 , Plutarque ne perd aucune occasion de présenter, comme l'écrit Ziegler ${ }^{34}$, des exemples de bienveillance, de bonté, de philanthrōpia ${ }^{35}$; cette dernière étant une vertu profondément enracinée dans son caractère, une vertu qu'il recommanda là où il la rencontra et dont il fit preuve personnellement et non seulement à l'égard de ses concitoyens et de toute créature à visage humain ${ }^{36}$. 10.6.

${ }^{30}$ Pour la $\varphi \imath \lambda \alpha v \theta \rho \omega \pi i ́ \alpha$ des anciens voir Plu., Quaest. conv. II 643D; Soll. an. 970A; Cim.

${ }^{31}$ Plu., Frat. am. 478C, 481F.

${ }^{32}$ Luc., XLI 9.

${ }_{33}^{3}$ Plu., Cat. Ma. 5.1.

${ }^{34}$ K. Ziegler, 1965, p. 367. Cf. J. De Romilly, 1979, p. 293: "la douceur ne semble pas, chez Plutarque, une notion qu'il auraît reçue toute faite, en héritage doctrinal, mais plutôt une valeur enracinée dans sa personalité et son caractère".

${ }^{35}$ Plu., Aem. 28. 1, 39. 9; Ages. 1. 5; Alex. 58. 8; Ant. 3. 10, 25. 3; Arist. 23. 1; Brut. 30.6 ; Caes. 34. 7 ; Cat. Mi. 21. 10, 23.1; 29.4; Cim. 6. 2, 10. 7; Cleom. 34. 3-4; Crass. 3. 5; Demetr. 4. 1,17.1, 50.1; Fab. 22.8 ; Comp. Phil. - Flam. 3. 4 ; Galb. 11.1, 20. 5 ; Luc. 18. 9, 29. 6; Marc. 1. 3, 10. 6, 20.1 (Marcel est le premier des Romains qui ait fait preuve de $\varphi 1 \lambda \alpha v \theta \rho \omega \pi i ́ \alpha) ;$ Pel. 21. 3; Per. 30. 3; Phoc. 5.1 ; Publ. 1.2, 4.5 ; Pyrrh. 11. 8 ; Sol. 15. 3 ; Thes. 6. 4, 36. 4. Mais à côté de ceux-ci il ne manque pas dans les Vies de Plutarque des exemples aussi d' $\alpha$ - $\varphi \imath \lambda \alpha v \theta \rho \omega \pi i ́ \alpha$ et d'ỏ̆ $\alpha v \theta \rho \omega \pi i ́ \alpha$ : Cat. Ma. 5.1, 5. 5; Dio 7.5 ; Nic. 11.2 ; Sull. 14.8.

${ }^{36}$ Voir Plu., Cat. Ma. 5.2: "nous ne devons pas traiter les êtres vivants comme des chassures ou des ustensiles, qu'on jette quand ils sont abîmés ou usés à force de servir, car il faut s'habituer à être doux et clément envers eux, sinon pour une autre raison, du moins pour s'exercer à la pratique de la vertu d'humanité ( $\varphi$ ı $\lambda \alpha v \theta \rho \omega \pi i ́ \alpha)$ ". 
Philanthrōpia ${ }^{37}$ dans l'acception commune d' "humanité" ou de "bienveillance" est un terme qui apparaît pour la première fois dans le monde grec dans la première moitié du $\mathrm{IV}^{\mathrm{e}}$ siècle av. J.-C. avec les Discours d'Isocrate et les Dialogues de Platon ${ }^{38}$, même si l'adjectif philanthrōpos, qui durant la période classique est l'un des trois termes "les plus couramment employés ... pour désigner la douceur" 39 , est déjà connu d'Eschyle qui l'utilise dans son

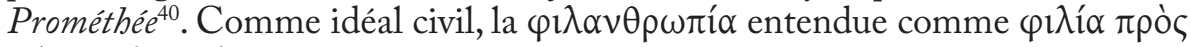

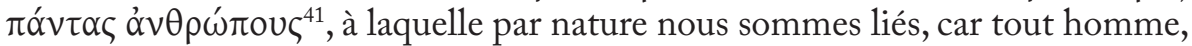

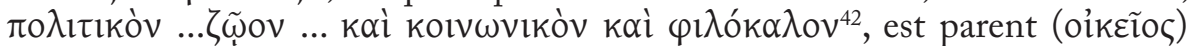

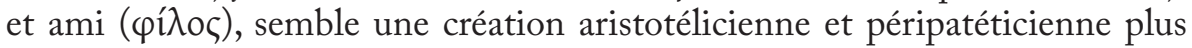
qu'une doctrine créée par les écoles de pensée modernes qui - en limitant la $\varphi \imath \lambda i ́ \alpha$ ou aux sages, comme le stoïcisme ancien, ou aux membres du groupe, comme l'épicuréisme - restèrent fondamentalement étrangères à cette philia pour l'homme en tant qu'homme. Après l'idéal cicéronien de l'bumanitas qui naît comme terme et comme concept au $\mathrm{I}^{\text {er }}$ siècle, en tant que plein exercice de la nature humaine, on assiste au début de l'époque impériale à la renaissance et à la diffusion du sentiment de $\varphi \imath \lambda \alpha v \theta \rho \omega \pi i ́ \alpha$ aussi bien chez les Grecs que chez les Romains comme bumanitas de la part d'intellectuels comme Philon, Sénèque, Épictète et Dion ; avec eux la philanthrōpia ne s'identifie plus avec le plein exercice de la nature humaine, mais elle parvient à devenir "expression du sentiment de la sociabilité" 43 .

Parmi les intellectuels des premiers siècles de l'époque impériale, Plutarque se révèle être l'auteur qui a fait un "usus frequentissimus" de la notion de philanthrōpia aussi bien dans les Euvres Morales que dans les Vies, où les occurrences sont bien plus nombreuses qu'a dénombrées Tromp de Ruiter dans la première moitié du $\mathrm{XX}^{\mathrm{e}}$ siècle dans une étude consacrée au sens et à l'emploi de ce terme $e^{44}$. Face aux 11 occurrences pour le substantif, 40 pour l'adjectif et 3 pour les formes adverbiales, que le chercheur a relevées dans tout l'opus de Plutarque (Euvres Morales et Vies), la banque de données du TLG signale 20 occurrences pour le substantif, 79 pour l'adjectif (dont 18 comme adjectif substantivé) et 16 pour l'adverbe dans les Euvres Morales, tandis que dans les Vies sont enregistrées 36 occurrences pour le substantif, 96 pour l'adjectif

37 Pour une définition générale de la notion de $\varphi \imath \lambda \alpha v \theta \rho \omega \pi i ́ \alpha$ voir D.L. III 98 Tñ

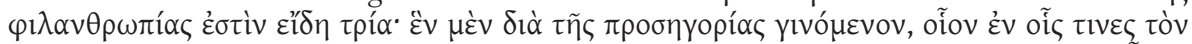

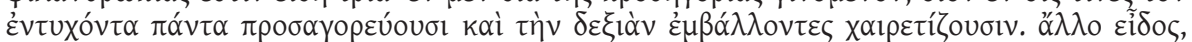

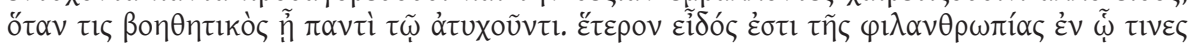

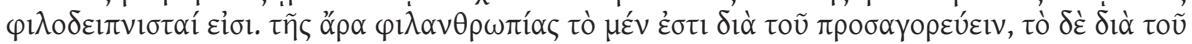

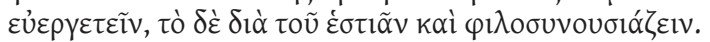

${ }^{38}$ Pour l'idée platonico-académicienne de $\varphi \imath \lambda \alpha v \theta \rho \omega \pi i ́ \alpha$ voir Pl., [Def.] 412E $\Phi \imath \lambda \alpha v \theta \rho \omega \pi i ́ \alpha$

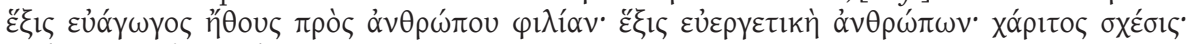
$\mu \vee \eta ́ \mu \eta \mu \varepsilon \tau ' \varepsilon \dot{\varepsilon} \varepsilon \gamma \varepsilon \sigma i ́ \alpha \varsigma$.

${ }_{39}$ J. De Romilly, 1979, p. 328; F. Frazier, 1996, p. 231.

${ }^{40}$ A., Pr. 11, 28.

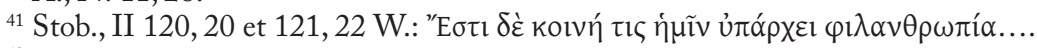

${ }^{42}$ Voir Asp., EN, CAG XIX 1, ed. G. Heylbut, Berolini 1889, p. 23, 7-8.

${ }^{43}$ Voir M. Pohlenz, 1978², pp. 125, 212.

${ }^{44}$ S. Tromp De Ruiter, 1932, pp. 297-9. 
(dont 22 comme adjectif substantivé) et 33 pour l'adverbe, pour un total de 280 occurrences environ pour les deux ouvrages ${ }^{45}$.

La notion de philanthrōpia ${ }^{46}$ au sein de l'opus plutarquien représente en général une qualité naturelle ou une aptitude de l'âme humaine susceptible d'être

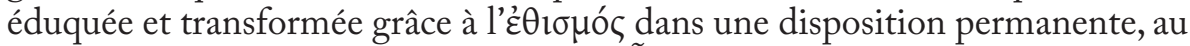
point de devenir une vertu du caractère ( $\tilde{\eta} \theta 0 \varsigma$ / habitus). Il s'agit d'un concept à la fois variable et adaptable, selon les circonstances, il se teint des couleurs

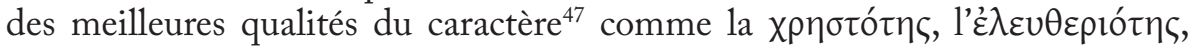

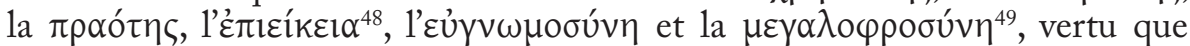
l'homme est appelé à mettre en pratique ne serait-ce que par humanité ${ }^{50}$. Mais cette qualité de l'âme ${ }^{51}$, que Romilly a définie comme une qualité "de sociabilité" ${ }^{52}$ et Frazier d' "humanité" ${ }^{33}$ se présente dans les Vies sous un aspect différent de celui qu'elle revêt dans les Euvres Morales. Dans les Vies, la philanthrōpia

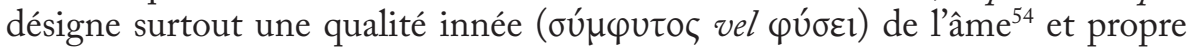
au peuple grec ${ }^{55}$ en général et athénien ${ }^{56}$ en particulier, tandis que dans les Euvres Morales, elle se présente le plus souvent comme une véritable vertu du caractère. En effet, dans les Vies, l'adjectif philanthrōpos se circonscrit plus

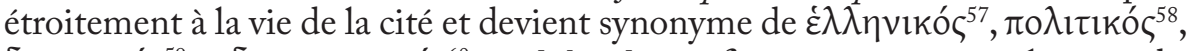

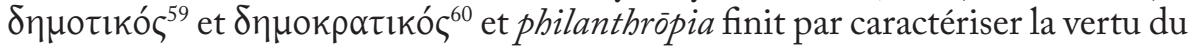

${ }^{45}$ Voir maintenant J.Ribeiro Ferreira, 2008, p. 89 n. 5.

${ }^{46}$ S. Tromp De Ruiter, 1932, pp. 287, 295, 299 : “apud Plutarchum

$\varphi \imath \lambda \alpha v \theta \rho \omega \pi i ́ \alpha \quad 11$ locis invenitur

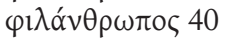

adverbialiter 3

... Plutarchi locis allatis satis apparere mihi videtur vocem et notionem philantropiae admodum florere apud illum auctorem".

${ }^{47}$ Voir J. Ribeiro Ferreira, 2008, pp. 78-84.

${ }^{48}$ Plu., Nic. 9. 6.

${ }^{49}$ Plu., Cap. ex inim. ut. 88C ; Alex. Magn. fort. virt. 336E..

${ }^{50}$ Plu., Cat. Ma. 5. 5. Pour la critique de Plutarque à la conduite de Caton l'Ancien, qui poussait la fidélité à ses principes d'austérité jusqu'à vendre ses esclaves, devenus vieux, pour ne pas avoir à les nourrir sans profit voir supra n.36.

${ }^{51}$ On pourrait définir la philanthrōpia avec les mêmes mots employés par le Stagirite pour

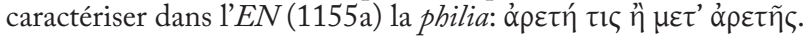

${ }^{52}$ J. De Romilly, 1979, p. 37.

${ }^{53}$ F. Frazier, 1996, p. 231.

${ }^{54}$ Voir Plu., Marc. 10. 6 . Mais "une nature généreuse et bonne, quand elle manque d'éducation, produit pêle-mêle des fruits excellents et des fruits détestables, comme un sol riche resté sans culture" (Plu., Cor. 1.3).

${ }^{55}$ Plu., Pelop. 6.5 ; Quaest. conv. II 643D.

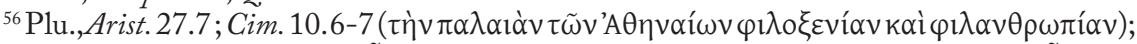

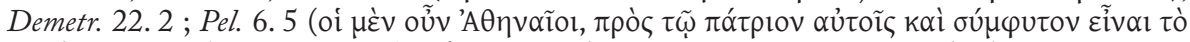

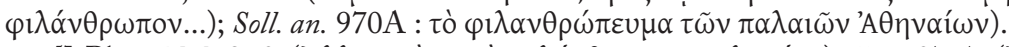

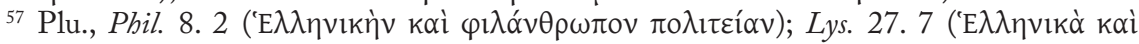
$\varphi \imath \lambda \alpha ́ v \theta \rho \omega \pi \alpha)$.

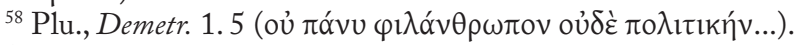

${ }^{59}$ Plu., Ages. 1. 5; Crass. 3. 5; Galb. 11.1; Oth. 1. 3.

${ }^{60}$ Plu., Comp. Cim.-Luc. 1, 6; Nic. 11, 2; Agis et Cleom. 34. 3. 


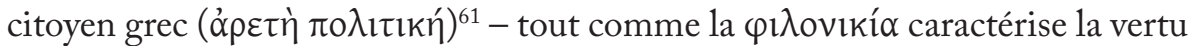

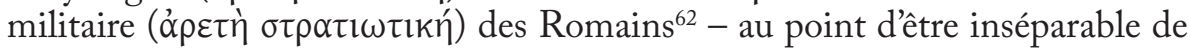
la notion de civilisation hellénique ${ }^{63}$. Cependant, dans les Euvres Morales la philanthrōpia, considérée comme étant $\varphi$ i $\lambda$ ía pour l'homme en tant qu'homme

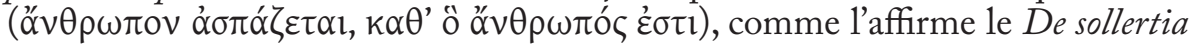
animalium $^{64}$, et assimilée à la $\varphi \imath \lambda$ ok $\alpha \lambda \chi_{1} \alpha^{65}$, se révèle être une vertu divine ou presque $^{66}$. Se modelant sur la perfection et sur l'amour que la divinité nourrit

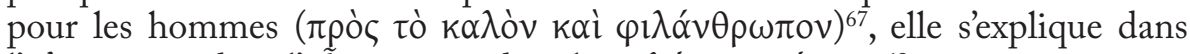

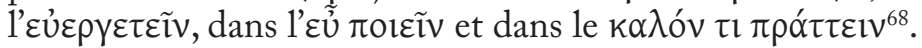

Convaincu de l'importance sociale et politique que revêt une telle vertu ${ }^{69}$, tournée vers l'extérieur au point d'embrasser l'humanité entière ${ }^{70}$ mais qui exige à son intérieur une éducation et une formation morale correcte, l'intellectuel de Chéronée ne perd pas une occasion de recommander comme seule $\delta 1 \delta \alpha \sigma \kappa \alpha \lambda i \alpha^{71}$, l'exercice ( $\left.\mu \varepsilon \lambda \varepsilon^{\varepsilon} \tau \eta\right)$ qui permet à la philanthrōpia de vertu naturelle qu'elle est à

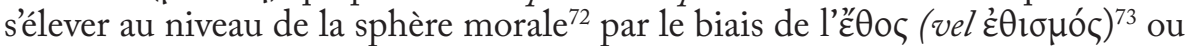
de la $\sigma u v \eta ́ \theta \varepsilon l \alpha^{74}$ de ces qui sont les premiers liens humains que les hommes ont

${ }^{61}$ Plu., Arist. 27. 7.

${ }^{62}$ Cf. Plu., Phil. 3. 1; Marc. 20.1.

${ }^{63}$ Voir l'analisi di H. M. Martin JR., 1961, p.167: "These threee concepts - philantrōpia, civilization, Hellenism - seem almost inseparabile for Plutarch”. Cf. D. Del Corno, 1982, p. 15.

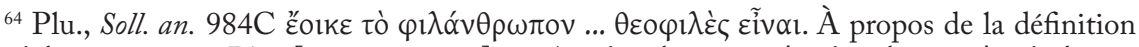

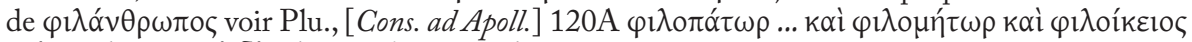

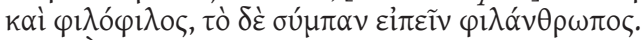

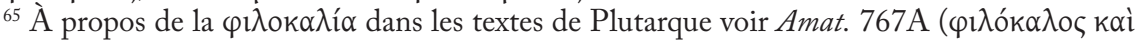

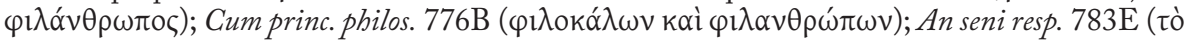

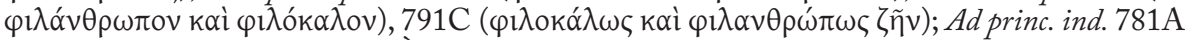

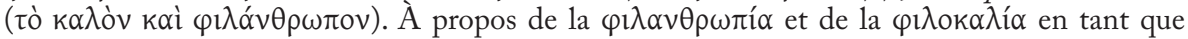

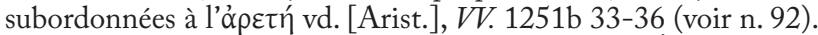

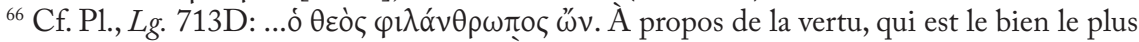
grand et le plus agréable voir Plu., Sol. 7. 2. À propos de la vertu comme le seul bien divin à la

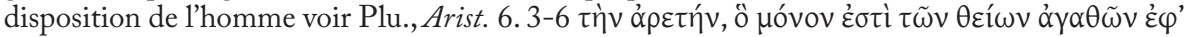
ทंน

${ }^{67}$ Plu., Ad princ. ind. 781A ; An seni resp. 786C.

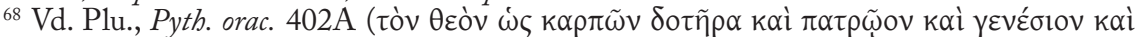

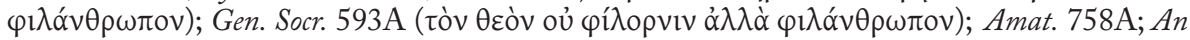
seni resp. 786B ; Stoic. rep. 1051E, $1052 \mathrm{~B}$.

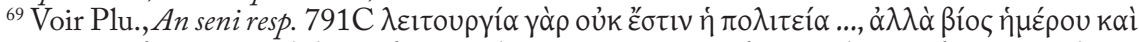

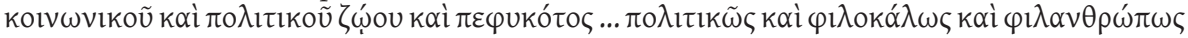
$\zeta \tilde{\eta} v$.

${ }^{70}$ Voir F. Frazier, 1996, p. 233: "la philanthrôpia , fidèle à son étymologie, est tournée vers l'extérieur et se dilate jusqu'à embrasser l'humanité entière”.

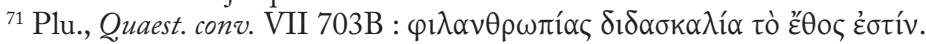

${ }^{72}$ Plu., Per. 1. 1; Virt. mor. 451E; Frat. am.482B.

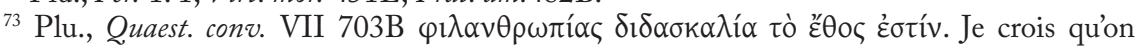
pourrait étendre à la $\varphi \imath \lambda \alpha v \theta \rho \omega \pi i ́ \alpha$ ce qu'écrit Plutarque à propos de la $\varphi \imath \lambda i ́ \alpha$ (Quaest. conv. IV

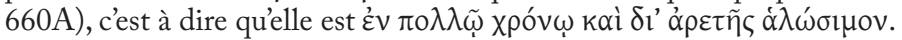

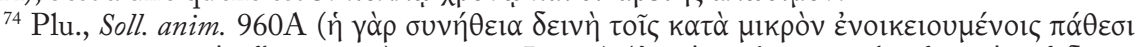

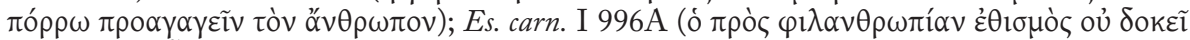

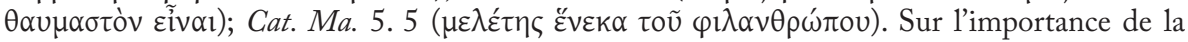




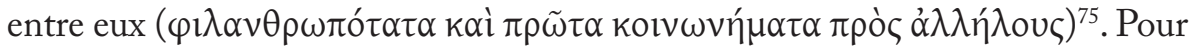
établir ces liens le banquet est un lieu privilégié ${ }^{76}$ : son but est moins de boire et

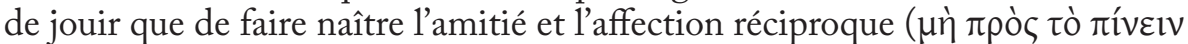

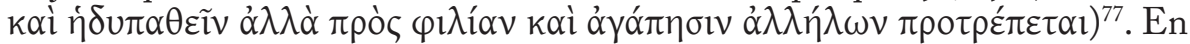
effet, dans le Septem sapientium convivium ${ }^{78}$ Plutarque affirme que l'homme judicieux (ó voṽv $\varepsilon^{\prime} \chi \omega V$ ) ne prend pas part à un banquet en se présentant comme un vase à remplir, mais pour trouver du plaisir à la conversation et dans le premier livre des Quaestiones convivales, il souligne le caractère élitiste et culturel du banquet, qui doit se tenir dans un climat de sobriété et de retenue,

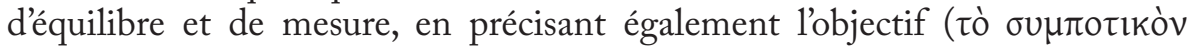

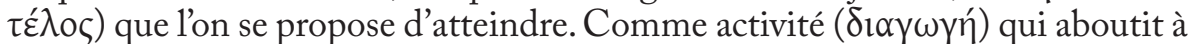
l'amitié ( le banquet vise à entraîner une amélioration du caractère ${ }^{81}$ et à consolider ou à

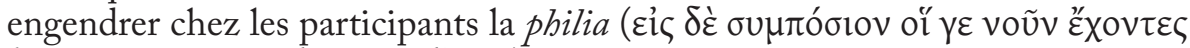

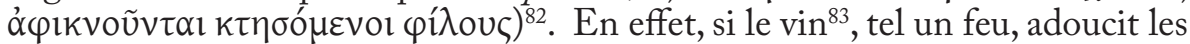
caractères et offre l'occasion d'établir des relations réciproques d'amitié ${ }^{84}$, c'est

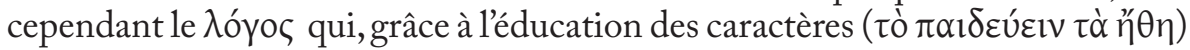

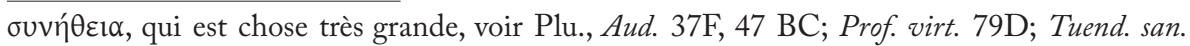

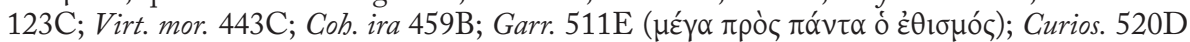

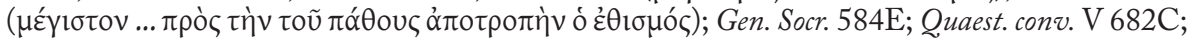
Soll. an. 959F. Plutarque toutefois ne surestime pas l'importance de la doctrine des Stoïciens

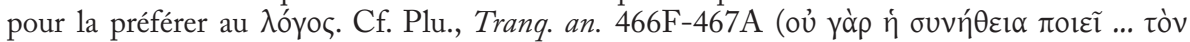

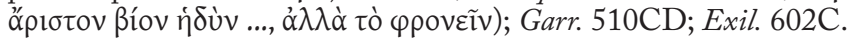

${ }^{75}$ Plu., Sept. sap. conv. $158 \mathrm{C}$.

${ }^{76}$ Sur le banquet dans son acception première de 'réunion conviviale' qui suit le banquet proprement dit et à propos du banquet comme l'un des événements les plus singuliers de la civilisation grecque, cf. M. Montanari, 1989, pp. 94-5; Plutarco, 1998, p. 14 sqq.

${ }_{77}$ Plu., Sept. sap. conv. 148AB; Quaest. conv. IV 660B (voir n. 86).

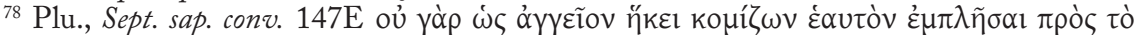

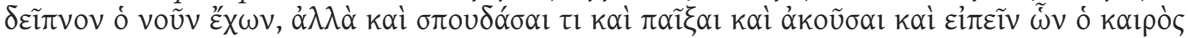

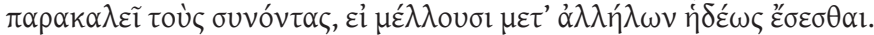

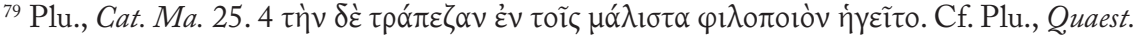
conv. II 632E.

${ }^{80}$ Plu., Quaest. conv. I 621C. Sur le rôle que joue la table pour faire naître la philanthropie

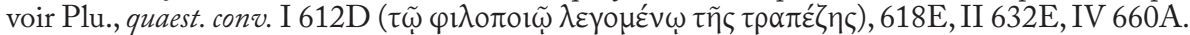

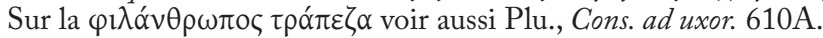

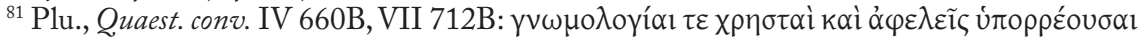

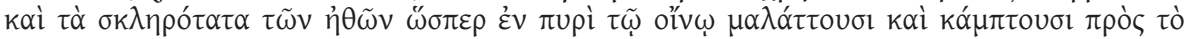

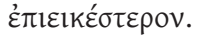

${ }^{82}$ Plu., Quaest. conv. IV 660A, I 621C (

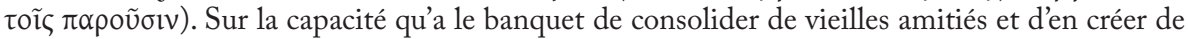

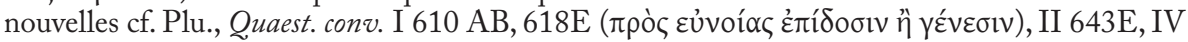
660B, 672E.

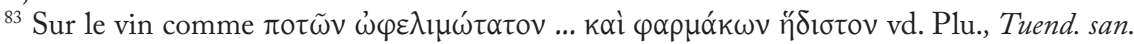
132B; Quaest. conv. III 647A, 655E.

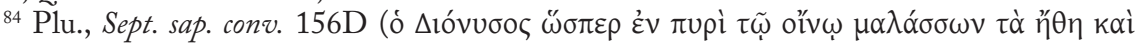

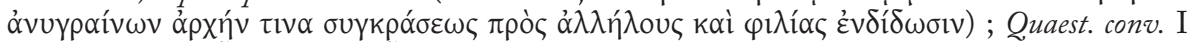

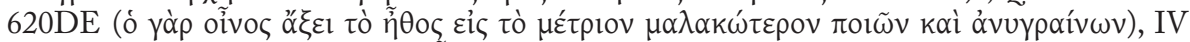

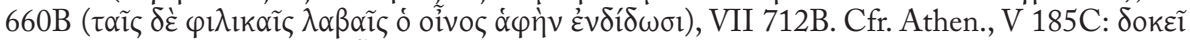

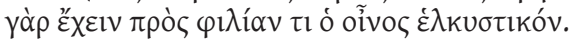




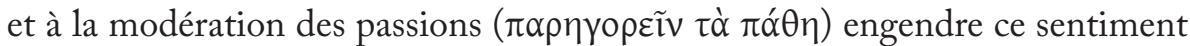
d'affection qui nous lie l'un à l'autre ( $\varphi$ ı $\dot{\alpha} \lambda \lambda \hat{n} \lambda$ ous $)^{85}$, c'est-à-dire la philanthrōpia ${ }^{86}$. Celle-ci, tout comme le bien ( $\tau$ ò $\kappa \alpha \lambda$ óv $^{87}$, attire à soi de manière active $(\pi \rho \alpha \kappa \tau i \kappa \tilde{\omega} \zeta)$, elle pousse tout de suite à l'action et, surtout, elle est capable de façonner le caractère $(\dot{\eta} \theta 0 \pi \text { oı ov })^{88}$.

Déterminer, à la lumière de ce que nous avons évoqué, les sources philosophiques de la doctrine plutarquienne de la philanthrōpia se révèle une entreprise assez difficile et encore moins sûre, car la reprise de cet idéal, comme nous l'avons vu, est - semble-t-il - moins le fruit d'une théorie élaborée dans le cadre d'une école de pensée spécifique, que la réponse personnelle et subjective à des exigences sociales qui, à cette époque-là, étaient en train de s'enraciner dans la conscience populaire ${ }^{89}$. Ayant exclu l'influence des écoles de pensée

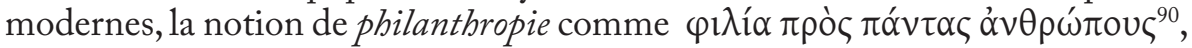

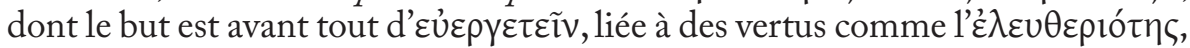

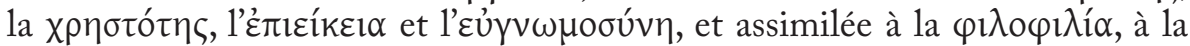
$\varphi 1 \lambda \circ \xi_{\varepsilon v i ́ \alpha}$ et notamment à la $\varphi \imath \lambda$ ok $\alpha \lambda \lambda_{1} \alpha$ se révèle une théorie qui trouve

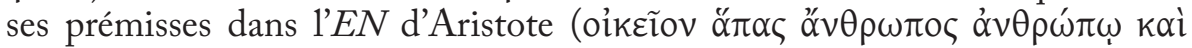
(í̂́ov.... $)^{91}$ - même si le Stagirite a recours à ce terme une fois seulement - et trouve correspondance dans un texte pseudo-aristotélicien, le De virtutibus et vitiis ${ }^{92}$ aussi bien que dans la doctrine théophrastienne de l'oỉkeló $\tau \eta \varsigma^{93}$, qui est différente de la doctrine stoïcienne de l'oíkeíwor ${ }^{94}$.

De tout façon ce que l'analyse des textes plutarquiens fait apparaître de manière très claire, c'est la confiance d'un éducateur qui, en conflit contre les écoles de pensée modernes, croit encore à l'existence d'autres rapports interpersonnels, sous-tendus par une grandeur d'âme et non pas dictés par

${ }^{85}$ Plu., Sept. sap. conv. $156 \mathrm{CD}$.

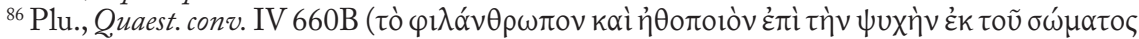

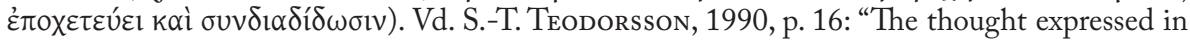
this sentence is curious indeed: 'Conversation channels and distributes by means of the wine its kindliness and characterforming influence from the body into the soul.' The assertion that wine has a character-forming action is curious, stille more that this is produced in the body and must be transported into the soul to be effective".

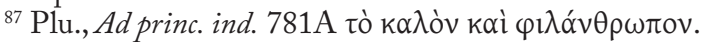

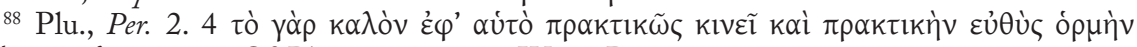

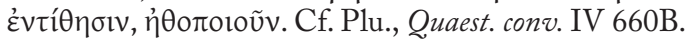

${ }^{89}$ Cf. J. De Romilly, 1979, p. 293.

${ }^{90}$ Stob., II 120, 20; 121, 22.

${ }^{91}$ Arist., EN1155a $20-22$.

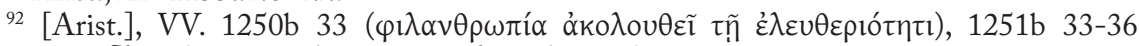

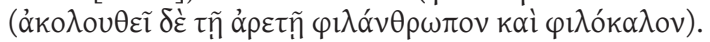

${ }_{93}$ Thphr., Frr. L 91, L 195-196 Fortenbaugh. Que Plutarque se soit inspiré de la doctrine

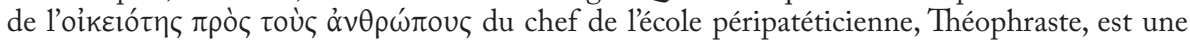
hypothèse qui est confirmée, semble-t-il, aussi dans le deuxième logos du De esu carnium. Voir G. Santese, 1999, p. 75: "Teofrasto fonda la communio iuris, il patto giuridico tra uomo e animale, sul concetto di oikkĩov, su un legame originario che lega tra loro i viventi”. Voir Plu., Frat. am. 428B et 490E = Thphr., Frr. L 96 et L 98 Fortenbaugh.

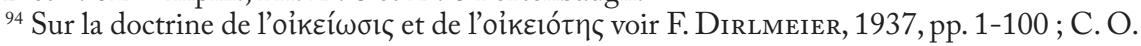
Brink, 1955; S. G. Pembroke, 1971; P. Moraux, 1973, p. 348; G. Striker, 1983. 
la justice ou par l'utilité, convaincu que bien vivre signifie aussi vivre avec un sentiment d'amitié et de communauté avec les autres ${ }^{95}$.

\section{BibLIOGRAPHIE}

Boudon-Millot, V. et Al., La science médicale antique. Nouveaux regards. Études réunies en l'honneur de J. Jouanna, Paris, 2008.

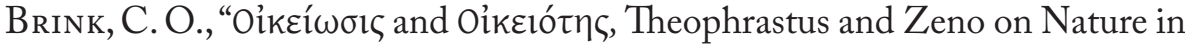
Moral Theory", Phronesis, 1 (1955) 123-45.

Del Corno, D., Il demone di Socrate. I ritardi della punizione divina, Milano, 1982.

Desideri, P., Dione di Prusa. Un intellettuale greco nell'impero romano, Messina-Firenze, 1978.

Dirlmeier,F., “Die Oikeiosis-Lehre Theophrasts”, Philologus Supplementband XXX (1937) 1-100.

Fialho, M. C., "Sócrates e a Paideia falhada de Alcibíades", in C. Soares et AL. (eds.), Ética e paideia em Plutarco, Coimbra, 2008, pp. 31-48.

Frazier, F., Histoire et morale dans les Vies Parallèles de Plutarque, Paris, 1996.

Jones, C. P., The Roman World of Dio Chrysostom, Harvard, 1978.

Martin Jr., H. M., "The concept of Philanthropia in Plutarch's Lives", AJPh, 82 (1961) 164-75.

Montanari, M., Convivio, vol. I, Bari, 1989, pp. 94-95.

Moraux, P., Der Aristotelismus bei den Griechen von Andronikos bis Alexander von Aphrodisias, Band I, Berlin, 1973.

Pembroke, S. G., "Oikeiōsis", in A. A. Long (ed.), Problems in Stoicism, London, 1971, pp. 114-49.

Pohlenz, M., La Stoa. Traduit en italien par O. De Gregorio, II, Firenze, $1978^{2}$.

Ribeiro Ferreira, J., "O doce afago da philanthropia", in C. Soares et al. (eds.), Ética e paideia em Plutarco, Coimbra, 2008, pp.85-97.

"Demotikos e Demokratikos na paideia de Plutarco", in C. SoAres Et AL. (eds.), Ética e paideia em Plutarco, Coimbra, 2008, pp. 69-84.

Romilly, J. de, La douceur dans la pensée grecque, Paris, 1979.

Santese, G., Il cibarsi di carne, Napoli, 1999.

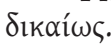

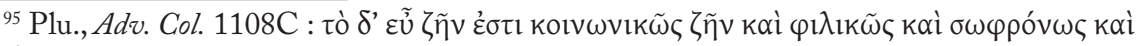


Scarcella, A. M., Plutarco. Conversazioni a tavola, I, (testo, traduzione e commento), A. Napoli, 1998.

Sirago, V. A., Involuzione politica e spirituale nell'impero del II secolo, Napoli, 1974.

Striker, G., "The Role of Oikeiosis in Stoic Ethics", OSAPh, 1 (1983) 14567.

Teodorsson, S.-T., A Commentary on Plutarch's Table Talks, vol. II (Books 4-6), Göteborg, 1990.

Tromp De Ruiter, S., "De vocis quae est $\varphi \imath \lambda \alpha v \theta \rho \omega \pi i ́ \alpha$ significatione atque usu”, Mnemosyne, 59 (1932) 271-306.

Ziegler, K., Plutarco (trad. it.), Brescia, 1965. 